\title{
Production of a unique pneumococcal capsule serotype belonging to serogroup 6
}

\begin{abstract}
Correspondence
Moon H. Nahm

nahm@uab.edu
\end{abstract}

Received 24 September 2008

Revised 4 November 2008

Accepted 12 November 2008

\author{
Preston E. Bratcher, ${ }^{1}$ In H. Park, ${ }^{2}$ Susan K. Hollingshead ${ }^{1}$ \\ and Moon H. Nahm ${ }^{1,2}$ \\ ${ }^{1}$ Department of Microbiology, University of Alabama at Birmingham, Birmingham, AL 35294, USA
${ }^{2}$ Department of Pathology, University of Alabama at Birmingham, Birmingham, AL 35294, USA
}

Serogroup 6 of Streptococcus pneumoniae contains three serotypes, named $6 \mathrm{~A}, 6 \mathrm{~B}$ and $6 \mathrm{C}$, with highly homologous capsule gene loci. The $6 \mathrm{~A}$ and $6 \mathrm{~B}$ capsule gene loci consistently differ from each other by only one nucleotide in the wciP gene. The $6 \mathrm{~A}$ capsule gene locus has a galactosyltransferase, which has been replaced with a glucosyltransferase in the $6 \mathrm{C}$ capsule gene locus. We considered that a new serotype named ' $6 \mathrm{X} 1$ ' would be possible if the galactosyltransferase of the $6 \mathrm{~B}$ capsule gene locus is replaced with the glucosyltransferase of $6 \mathrm{C}$. We demonstrate that this gene transfer yields a viable pneumococcal strain and that the capsular polysaccharide (PS) from this strain has the predicted chemical structure and serological similarity to the capsular PS of the $6 B$ serotype. The new strain (i.e. serotype $6 \mathrm{X} 1$ ) is typed as $6 \mathrm{~B}$ by the quellung reaction, but it can be distinguished from $6 \mathrm{~B}$ strains with mAbs to $6 \mathrm{~B}$ PS.

Reexamination of 264 pneumococcal isolates that had been previously typed as $6 \mathrm{~B}$ with classical typing methods revealed no isolates expressing serotype 6X1. Nevertheless, this study shows that this capsular PS is biochemically possible and could exist/emerge in nature.

\section{INTRODUCTION}

Streptococcus pneumoniae is a major human pathogen commonly responsible for pneumonia, bacteraemia, meningitis and otitis media, especially among young children and older adults (Fedson, 1988). The most prominent virulence factor of the pneumococcus is the capsular polysaccharide (PS), which coats the surface of the bacterium to block antibodies and complement from binding to surface moieties and being recognized by phagocytic cells (Avery \& Dubos, 1931). To avoid host immunity, S. pneumoniae, as a species, can express at least 91 distinct capsules which are chemically and serologically distinct (Henrichsen, 1995; Park et al., 2007b). Antibodies against this PS have been shown to provide serotypespecific protection from infection, and vaccines against pneumococcus incorporate capsular PSs of the most prevalent strains (Cole, 1913). For example, serogroup 6 strains are very common in invasive pneumococcal disease and the current vaccines are formulated to protect against serogroup 6 infections (Hausdorff et al., 2000).

Serogroup 6 has classically contained two serotypes, $6 \mathrm{~A}$ and 6B (Kamerling, 2000). These two serotypes produce capsules with very similar structures. Both of these PSs have repeating units composed of galactose-glucose-

Abbreviation: PS, polysaccharide.

The GenBank/EMBL/DDBJ accession number for the TIGR6X1 sequence of Streptococcus pneumoniae is EU714777. rhamnose-ribitol-phosphate, but the linkage between rhamnose and ribitol is $1 \rightarrow 3$ for $6 \mathrm{~A}$ and $1 \rightarrow 4$ for $6 \mathrm{~B}$ (Kamerling, 2000). The two serotypes also have very similar capsule gene loci, which are about $17 \mathrm{~kb}$ in size and contain all the genes for capsule biosynthesis (Jiang et al., 2001; Park et al., 2007a). The only genetic difference between $6 \mathrm{~A}$ and $6 \mathrm{~B}$ serotypes has been attributed to one nucleotide of $w c i P$, which encodes a rhamnosyltransferase responsible for the rhamnose-ribitol linkage (Aanensen et al., 2007; Mavroidi et al., 2004). WciP with a codon for serine at residue 195 is associated with serotype $6 \mathrm{~A}$, but a codon for asparagine is associated with 6B (Mavroidi et al., 2004).

Recently, a new serotype, 6C, was discovered using two mAbs (Lin et al., 2006; Park et al., 2007b). Although 6C was previously typed as $6 \mathrm{~A}$ with classical serotyping tools, chemical analysis revealed that the galactose of the 6A PS is replaced by a glucose residue in $6 \mathrm{C}$. Genetic studies of the $6 \mathrm{~A}$ and $6 \mathrm{C}$ capsule gene loci have shown that wciN is responsible for the difference, as substitution of $w c i N_{6 C}$ (wciN of 6C) for $w c i N_{6 A}(w c i N$ of $6 \mathrm{~A}$ ) through homologous recombination results in a serotype switch from $6 \mathrm{~A}$ to $6 \mathrm{C}$ (Park et al., 2007a).

The discovery of $6 \mathrm{C}$ led to the logical suggestion that recombination might also produce a new member of the serogroup 6 family, herein labelled ' $6 \mathrm{X1}$ ', which would have $w c i P$ of $6 \mathrm{~B}$ and $w c i N_{6 C}$. The designation $6 \mathrm{X} 1$ is used to distinguish this artificial serotype from the next naturally 
found serogroup 6 member, which, if discovered, might be designated 6D. Chemically, 6X1 PS may have glucose instead of galactose and have a $1 \rightarrow 4$ rhamnose-ribitol linkage. However, it was not clear whether the theoretical $6 \mathrm{X} 1$ serotype was indeed biologically feasible, nor was it clear whether it might already exist in nature. Therefore, we have produced a serotype 6X1 strain and examined our laboratory collection of pneumococcal isolates for a possible $6 \mathrm{X} 1$ strain.

\section{METHODS}

Bacterial strains and culture. To determine whether $6 \mathrm{X} 1$ exists in nature, 264 pneumococcal isolates that were previously serotyped as ' $6 \mathrm{~B}$ ' by classical means were reserotyped for serotypes $6 \mathrm{~B}$ or $6 \mathrm{X} 1$ using mAbs. The isolates were a part of our laboratory collection of $6 \mathrm{~B}$ isolates, which have originated from Africa, Asia, Australia, South America, North America and Europe. In addition to these, TIGR6A, TIGR6AX and TIGR6C, which are isogenic strains of TIGR4 expressing the 6A-type capsule, no capsule, and 6C-type capsule (Park et al., 2007a), were used as assay controls or as a source of DNA. The source of the TIGR4 strain was the authors' laboratory (Tettelin et al., 2001). Additional TIGR4 variants, TIGR6B, TIGR6BX and TIGR6X1, were prepared as described below. All bacteria were grown in Todd-Hewitt broth (BD Biosciences) supplemented with $0.5 \%$ yeast extract (THY) and kept frozen at $-80{ }^{\circ} \mathrm{C}$ until used. The TIGR6X1 strain will be available upon request from the University of Alabama at Birmingham (UAB) to all qualified investigators for research purposes.

PCR and DNA sequencing. PCR mixtures contained $38.8 \mu \mathrm{l}$ sterile water, $2 \mu \mathrm{l}$ of each primer $\left(5 \mathrm{pmol} \mu \mathrm{l}^{-1}\right), 2 \mu \mathrm{l} 10 \mathrm{mM}$ dNTP, $5 \mu \mathrm{l}$ $10 \times$ LA Taq buffer solution (Takara Biochemical), and $0.2 \mu \mathrm{l}$ LA Taq polymerase (2.5 $\mathrm{U} \mathrm{\mu l}^{-1}$; Takara Biomedical). As template, either chromosomal DNA isolated with a Wizard genomic DNA purification kit (Promega) or colonies grown on blood agar plates were used. Thermal cycling conditions varied depending on the primer set used. PCR products were analysed by electrophoresis in $1 \%$ agarose gels. The primers used are listed in Table 1. PCR products were purified using the Wizard PCR Clean-up System (Promega), and the DNA sequencing was performed by the Genomics Core Facility at UAB. DNA sequences were analysed with Lasergene v5.1 software (DNASTAR) and were compared with the previously reported sequences of the $6 \mathrm{~B}$ and 6C cps loci in GenBank (accession nos CR931639 and EF538714, respectively) (Mavroidi et al., 2004).

Construction of TIGR6X1 by replacing $\operatorname{wciN}_{6 A}$ of TIGR6B with $\boldsymbol{w c i N}_{\mathbf{6 c}}$. Our strategy for creating TIGR6X1 is described in Fig. 1. First, TIGR6B expressing serotype $6 \mathrm{~B}$ was prepared by inserting the $6 \mathrm{~B}$ capsule gene locus region into the TIGR4 genetic background using the Janus-cassette system, as previously described in detail (Park et al., 2007a). Second, the wciN gene was removed from TIGR6B (Park et al., 2007a) by transforming it with cassette 1 and selecting for kanamycin-resistant isolates. Cassette 1 has the Janus cassette, which contains a kanamycin-resistance gene $\left(k a n A^{R}\right)$ and a streptomycinsensitivity gene $\left(r p s L^{+}\right)$, and two flanking regions designed for homologous recombination to the $6 \mathrm{~B}$ capsule gene locus (Park et al., 2007a). A kanamycin-resistant strain was obtained and back-crossed into TIGR6B three times using genomic DNA from this resistant strain. Back-crossing was performed in order to minimize the possibility of unwanted mutations in the TIGR6B background. The resulting strain, which was labelled TIGR6BX, lost wciN and did not produce capsular PS. To insert $w_{c i N_{6 C}}$, TIGR6BX was transformed with Cassette 2. Cassette 1 and cassette 2 were prepared from the genomic DNAs of TIGR6AX and CHPA388, respectively, using primer set 5113 and 3102 (Park et al., 2007a). While Cassette 2 contained a part of $w c i P$ in addition to $w c i N_{6 C}$, it did not contain the $w c i P$ codon responsible for distinguishing $6 \mathrm{~A}$ and $6 \mathrm{~B}$ serotypes (Fig. 1). After selection for streptomycin resistance and back-crossing against TIGR6BX three times, a streptomycin-resistant strain was produced, designated TIGR6X1. When the capsule gene locus of TIGR6X1 was sequenced from wchA to wciP, the sequence showed that $w c i N_{6 B}$ had been replaced with the $w c i N_{6 C}$ gene, as intended. For this sequencing, primer sets 5114-3141, 5138-3104 and 5106-3105 were used to produce amplicons, and primers 5103, 5108 and 5129 were used in sequencing (see Table 1 for primer sequences). The TIGR6X1 sequence has been deposited in GenBank (accession no. EU714777). TIGR6X1 was morphologically indistinguishable from TIGR6B when grown on blood agar plates. Also, TIGR6X1 grew as well as other pneumococcal strains in THY broth (data not shown).

Quellung reaction. Bacterial colonies from blood agar plates were suspended in a small volume of PBS $(0.137 \mathrm{M} \mathrm{NaCl}, 2.31 \mathrm{mM}$ $\mathrm{KH}_{2} \mathrm{PO}_{4}, 7.69 \mathrm{mM} \mathrm{Na} \mathrm{HPO}_{4}$ and $2.15 \mathrm{mM} \mathrm{KCl}$ ), and $2 \mu \mathrm{l}$ of this broth was combined with $2 \mu \mathrm{l}$ serum and $2 \mu \mathrm{l}$ methylene blue dye solution $\left(3 \mathrm{mg} \mathrm{ml}^{-1}\right.$ methylene blue, $1.5 \mathrm{mg} \mathrm{ml}^{-1} \mathrm{NaCl}$ in sterile water) on a glass microscope slide. After adding a coverslip, mixtures were examined under bright-field microscopy using a $\times 100$

Table 1. List of PCR primers

\begin{tabular}{|c|c|c|}
\hline Primer & Sequence $\left(5^{\prime}-3^{\prime}\right)$ & Source or reference \\
\hline \multicolumn{3}{|c|}{ Forward primers } \\
\hline 5103 & AAACATGACATCAATTACA & Park et al. (2007a) \\
\hline 5106 & TACCATGCAGGGTGGAATGT & Park et al. (2007a) \\
\hline 5108 & ATGGTGAGAGATATTTGTCAC & Park et al. (2007a) \\
\hline 5113 & GGGAAAAATAAAAAATAGGTCGGG & Park et al. (2007a) \\
\hline 5114 & TTAGTGACGGAGGCAGGTGAA & This study \\
\hline 5129 & TCCTACTTACAGCAACTTCTCGTG & This study \\
\hline 5138 & AAAGCTATGTCGCCTGCTAAAAAAGCAATGGCTA & This study \\
\hline \multicolumn{3}{|c|}{ Reverse primers } \\
\hline 3102 & CTGGCATGTCATCTTTAGAAAA & This study \\
\hline 3104 & CCTGAAAACAATACTACTT & This study \\
\hline 3105 & TTCССАТСТСТАААСАТТСТССТ & This study \\
\hline 3141 & GGCGACATAGCTTTTCTTTCAATATCTT & This study \\
\hline
\end{tabular}




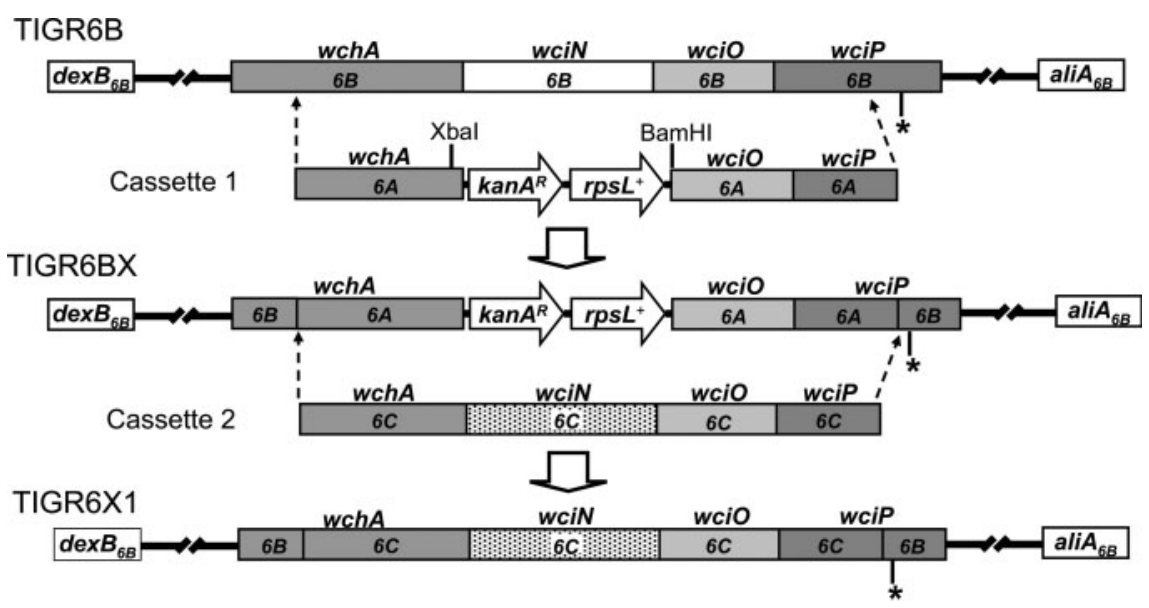

Fig. 1. Diagram of the wciN region exchange experiment; an asterisk at the wciP gene indicates the critical codon (serine versus asparagine) associated with the 6A/6B serotype difference (Mavroidi et al., 2004). kan $A^{R}$, kanamycin-resistance gene; $r p s L^{+}$, streptomycin-sensitivity gene.

oil-immersion lens. The rabbit antisera specific for serotypes $6 \mathrm{~A}$ and $6 \mathrm{~B}$ were prepared by the Centers for Disease Control and Prevention (CDC). Factor 6a serum made by Staten Seruminstitut was purchased from Mira Vista Diagnostics.

Inhibition ELISA to distinguish serotypes $6 B$ and $6 \times 1$. The two serotypes were distinguished using an inhibition-type ELISA. Briefly, the wells of ELISA plates (Corning Costar) were coated at $37{ }^{\circ} \mathrm{C}$ with $5 \mu \mathrm{g} \mathrm{ml}^{-1} 6 \mathrm{~B}$ capsular PS (ATCC) overnight in PBS. After washing the plates three times with PBS containing $0.05 \%$ Tween 20, $50 \mu \mathrm{l}$ of a previously diluted bacterial culture supernatant (or lysate) was added to the wells along with $50 \mu \mathrm{l}$ anti-6B mAb. Pneumococcal lysates were prepared by growing pneumococci overnight in $1 \mathrm{ml}$ THY broth without shaking and then incubating the tubes for $15 \mathrm{~min}$ at $37{ }^{\circ} \mathrm{C}$ with a lysis buffer $(0.1 \%$ sodium deoxycholate, $0.01 \%$ SDS, $0.15 \mathrm{M}$ sodium citrate in deionized water). Culture supernatants of $6 \mathrm{~B}$-specific hybridomas Hyp6BM7 and Hyp6BM8 were used at dilutions of $1: 50$ and 1:100, respectively. These hybridomas were produced from a fusion of myeloma cells with spleen cells isolated from mice immunized with 6B PS (Sun et al., 2001). After $30 \mathrm{~min}$ incubation in a humid incubator at $37{ }^{\circ} \mathrm{C}$, the plates were washed three times and incubated for $30 \mathrm{~min}$ with alkaline phosphatase-conjugated goat anti-mouse immunoglobulin (Sigma). The plates were washed three times, and then $100 \mu \mathrm{l}$ paranitrophenyl phosphate substrate (Sigma) in diethanolamine buffer at a concentration of $1 \mathrm{mg} \mathrm{ml}^{-1}$ was added, and allowed to incubate at room temperature for $1-2 \mathrm{~h}$. The $A_{405}$ was read with a microplate reader (BioTek Instruments).

Purification of capsular PS. Capsular PS expressing serotype 6X1 was purified in two different ways. One method was to purify the PS by ethanol precipitation, ion-exchange chromatography and molecular mass-sizing chromatography, as described previously (Park et al., 2007b). The other method, which is faster than the first method, was to purify capsular PS after removing protoplasts, as described below. TIGR6X1 was grown in 11 THY broth without shaking until the culture reached $\mathrm{OD}_{600} \sim 0.4$. The culture was then centrifuged at $15000 \mathrm{~g}$ for $10 \mathrm{~min}$. The cell pellet was washed twice with $11 \mathrm{PBS}$, resuspended in $30 \mathrm{ml}$ protoplast buffer [ $20 \%$ sucrose, $5 \mathrm{mM}$ Tris/ $\mathrm{HCl}$ (pH 7.4), $2.5 \mathrm{mM} \mathrm{Mg}_{2} \mathrm{SO}_{4}$ in deionized water] with mutanolysin (Sigma) at a concentration of $20 \mathrm{U} \mathrm{ml}^{-1}$, and allowed to incubate overnight at room temperature. The next day, the bacterial cells were examined under a phase-contrast microscope to ensure that 'protoplasting' had occurred, and then protoplasts were removed by centrifugation at $27000 \mathrm{~g}$ for $15 \mathrm{~min}$. The supernatant was sterilized through a $0.22 \mu \mathrm{m}$ pore-size filter, diluted $1: 1$ in deionized water, and loaded onto a DEAE-Sepharose column (Amersham Biosciences) with a $2 \mathrm{ml}$ bed volume. The column was washed with $4 \mathrm{ml} 50 \mathrm{mM}$ ammonium acetate, and the PS was eluted from the column with $4 \mathrm{ml} 500 \mathrm{mM}$ ammonium acetate. After lyophilization, the eluted PS was loaded on a Sephacryl S-300 HR column (Amersham Biosciences) with a bed volume of $130 \mathrm{ml}$ and the PS was eluted with $10 \mathrm{mM}$ Tris/ $\mathrm{HCl}(\mathrm{pH}$ 7.4). The fractions were tested for the presence of PS by an inhibition assay using Hyp6BM8. The first $10 \mathrm{ml}$ of fractions, which contains most of the PS, were pooled and lyophilized.

Monosaccharide composition analysis of PS. A $1 \mathrm{mg}$ sample of lyophilized capsular PS prepared by the protoplast method was dissolved in $500 \mu \mathrm{l}$ of $1 \mathrm{M} \mathrm{HCl}$ and incubated at $80^{\circ} \mathrm{C}$ for $16 \mathrm{~h}$. After drying the sample under a nitrogen stream, the remaining PS was washed twice with $250 \mu \mathrm{l}$ methanol. The sample was then incubated with $200 \mu \mathrm{l}$ Tri-Sil Reagent (Pierce Biotech) to trimethylsilylate all the monosaccharides. The reaction products were analysed on a gasliquid chromatograph/mass spectrometer (Varian 4000) fitted with a $15 \mathrm{~m}$ ( $0.25 \mathrm{~mm}$ diameter) VF-5 capillary column. Column temperature was maintained at $100{ }^{\circ} \mathrm{C}$ for $5 \mathrm{~min}$ and then increased to $275{ }^{\circ} \mathrm{C}$ at $20{ }^{\circ} \mathrm{C} \mathrm{min}{ }^{-1}$, and finally held at $275^{\circ} \mathrm{C}$ for $5 \mathrm{~min}$. The effluent was analysed by MS using the electron impact ionization mode. The area of each monosaccharide peak in GLC-MS was determined using Varian MS Workstation v6.5 software.

Analysis of PS by MS-MS. Intact capsular PSs prepared by the ethanol-precipitation method were hydrolysed to their repeating units before analysis by MS. A $2 \mathrm{mg}$ sample of PS was hydrolysed in $1 \mathrm{ml}$ $10 \mathrm{mM} \mathrm{NaOH}$ at $85{ }^{\circ} \mathrm{C}$ for $120 \mathrm{~h}$, followed by another hydrolysis with $50 \mathrm{mM} \mathrm{NaOH}$ at $85{ }^{\circ} \mathrm{C}$ for $120 \mathrm{~h}$. At the end of hydrolysis, all samples were neutralized with $0.1 \mathrm{M} \mathrm{HCl}$.

MS-MS was performed at the Mass Spectrometry Shared Facility at the UAB with a Micromass Q-TOF2 mass spectrometer equipped with an electrospray ion source. The samples, dissolved in distilled water, were injected into the mass spectrometer with running buffer (50:50 acetonitrile : water containing $0.1 \%$ formic acid) at a rate of $1 \mu \mathrm{min}^{-1}$ using a Harvard syringe pump. The injected sample was negatively ionized with electrospray and detected with a time-of-flight 
mass spectrometer. For MS-MS, the parent ion was fragmented into daughter ions by energizing it to either 35 or $40 \mathrm{eV}$ before collision with argon gas. The daughter ions were analysed with a time-of-flight mass spectrometer. The MS-MS spectra were processed using the Max-Ent3 module of MassLynx 2.5.

Oxidation and reduction of PS. Capsular PSs were dissolved in $80 \mathrm{mM}$ sodium acetate buffer $(\mathrm{pH} \mathrm{4})$ at a concentration of $1 \mathrm{mg}$ $\mathrm{ml}^{-1}$. Sodium periodate was added to the PS solution to a final concentration of $40 \mathrm{mM}$ and the reaction mixture was incubated in the dark at $4{ }^{\circ} \mathrm{C}$ for $72 \mathrm{~h}$. Excess periodate was destroyed by adding ethylene glycol. To determine the intact monosaccharides of the oxidized capsular PS, PS was then lyophilized and analysed using GLC-MS as described above. To investigate the glycosidic bonds, the sample was reduced with sodium borohydride or sodium tetradeuteroborate as previously described (Park et al., 2007b), before being subjected to MS-MS as described above. 6X1 PS was prepared by the protoplast method, and 6B PS was obtained from the ATCC.

Hydrolytic stability assay. A $0.9 \mathrm{ml}$ volume of PS $\left(2 \mathrm{mg} \mathrm{ml}^{-1}\right)$ in water was mixed with $0.1 \mathrm{ml} 0.1 \mathrm{M} \mathrm{NaOH}$, and this solution was split into two Eppendorf tubes and incubated at $85{ }^{\circ} \mathrm{C}$. At the indicated times, $0.1 \mathrm{ml}$ was removed from these samples, neutralized with $0.1 \mathrm{M} \mathrm{HCl}$, and then stored at $4{ }^{\circ} \mathrm{C}$ until used in the inhibition ELISA. Using the same buffers and incubation conditions described for the inhibition ELISA above, plates were coated with $100 \mu \mathrm{l} 6 \mathrm{~A}, 6 \mathrm{~B}$, $6 \mathrm{C}$ or $6 \mathrm{X} 1 \mathrm{PS}\left(5 \mu \mathrm{g} \mathrm{m}{ }^{-1}\right)$. The ELISA was performed with the hydrolysed samples on plates coated with their respective PSs. For 6A and 6C PSs, Hyp6AG1 was used as the primary antibody (as performed in Park et al., 2007b), and for 6B and 6X1, Hyp6BM8 was used (as described above). Data shown are the average of samples run in duplicate.

\section{RESULTS}

\section{X1 capsular PS is structurally different from 6B capsular PS}

Recently, we have demonstrated the structure of $6 \mathrm{C}$ capsular PS by identifying its monosaccharide composition by GLC-MS, and identifying the sequence of monosaccharides and their glycosidic linkages by MS-MS (Park et al., 2007b). We used similar approaches to show that TIGR6X1 produces a capsular PS that is chemically different from that of TIGR6B. We first determined the monosaccharide/ribitol composition of TIGR6B and TIGR6X1 capsular PS by GLC-MS. The chromatogram for 6B showed peaks for ribitol, rhamnose, galactose and glucose, as expected for 6B capsular PS (Fig. 2). However, the 6X1 chromatogram did not show galactose peaks, although it showed peaks for ribitol, rhamnose and glucose (Fig. 2). Thus, TIGR6X1 produces a capsular PS different from that of TIGR6B. We then analysed the monosaccharide composition after treating TIGR6B and TIGR6X1 PSs with sodium periodate, which selectively destroys ribitol and monosaccharides with vicinal glycols. As expected for 6B PS, periodate treatment of TIGR6B PS eliminated the peaks for ribitol and galactose (Fig. 2), but the treatment did not alter the rhamnose and glucose peaks. In contrast, periodate treatment of TIGR6X1 PS extinguished the ribitol peak and reduced the glucose peaks, such that the glucose peak area became similar to the rhamnose peak

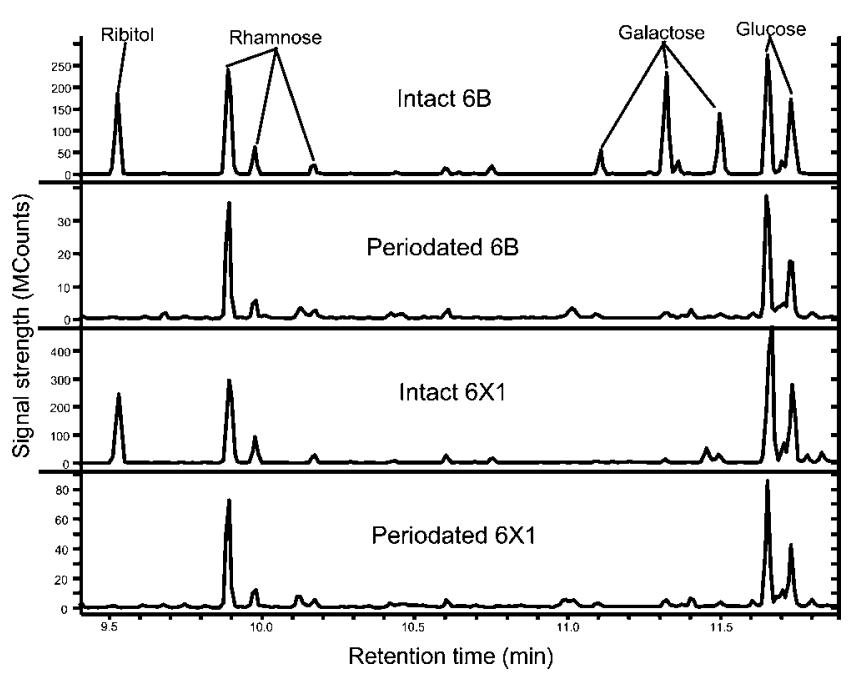

Fig. 2. GLC-MS chromatograms showing carbohydrate composition of capsular PS from pneumococcal strains expressing 6B and 6X1 serotypes and before and after periodate treatment. The monosaccharides are identified in the top chromatogram. Each panel is labelled with a description of the sample.

area. This suggested that the repeating unit of $6 \mathrm{X} 1 \mathrm{PS}$ has two glucose residues, whereas the repeating unit of $6 \mathrm{~B}$ PS has one glucose residue and one galactose residue.

To determine whether the monosaccharide sequence of the 6X1 PS is as proposed in Fig. 3(a), we performed an alkali hydrolysis which breaks the phosphodiester bonds and produces repeating units. As previously observed for 6C PS (Park et al., 2007b), the hydrolysis yields two types of repeating units of identical mass, one with the phosphate ion linked to ribitol (labelled forward fragmentation) and another linked to glucose (labelled reverse fragmentation). The phosphate ion endows the repeating unit with a negative charge. When the alkali hydrolysis product was analysed for negative ions by MS-MS, the results showed two prominent peaks of 683 and 701 amu (Fig. 3b), which were identical to the anhydrous and hydrated masses, respectively, of the predicted repeating unit of 6X1 PS (Fig. 3a). The peak at 260.902 amu was absent in other MSMS attempts and may represent a contaminant.

We then subjected the ion of 683 amu (i.e. the intact repeating unit) to argon collision and identified its daughter ions by MS-MS analysis. We found daughter ions at 521, 359 and $213 \mathrm{amu}$, which respectively represent daughter ions that have lost the first glucose, the second glucose and the rhamnose (Fig. 3c). Also we observed peaks at 549, 403 and $241 \mathrm{amu}$, which also correspond to the daughter ions formed after reverse fragmentation by losing ribitol, ribitol-rhamnose and ribitol-rhamnose-glucose 2, respectively (Fig. 3c). Three peaks with 113, 127 and 145 amu were absent in other MS-MS analyses and may represent contaminants. Thus, the monosaccharide sequence of the 6X1 PS repeating unit is glucose 1-glucose 
(a)

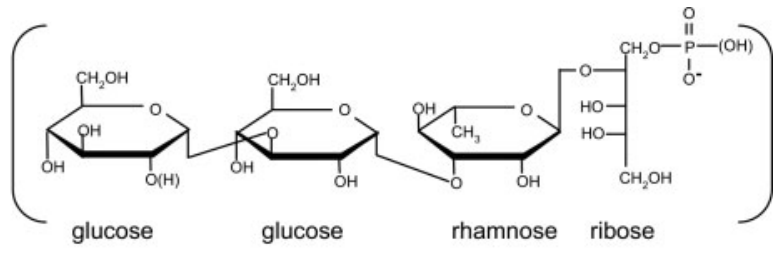

(b)
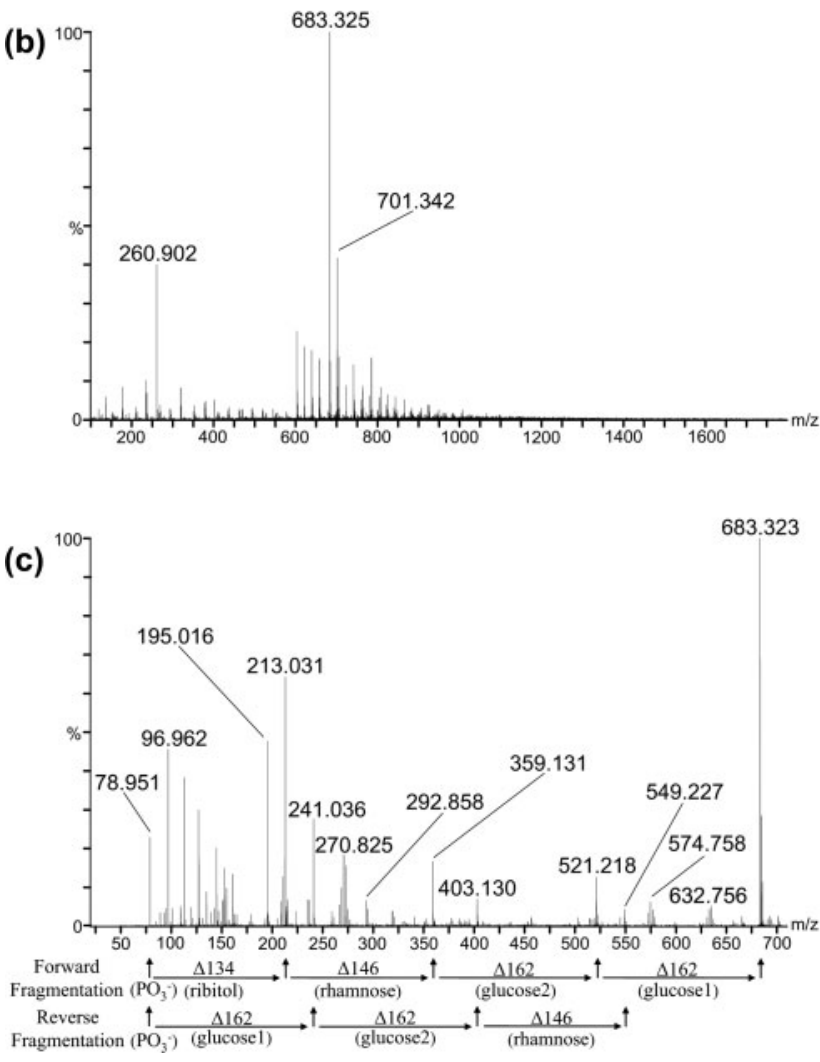

Fig. 3. (a) Proposed structure of the hydrated form of the repeating unit of $6 \mathrm{X} 1$ capsular PS. The calculated molecular mass is $701 \mathrm{amu}$. (b) Mass spectrum of the repeating units. The peaks at $683.3 \mathrm{~m} / \mathrm{z}$ and $701.3 \mathrm{~m} / \mathrm{z}$ correspond, respectively, to the anhydrous and hydrated forms of the repeating units. (c) Daughter ions of the ion of 683.3 amu shown in (b). Daughter ions are identified at the bottom. The peaks at $270.825,574.758$ and $632.756 \mathrm{amu}$ and their satellite peaks (separated by $2 \mathrm{amu}$ due to chloride isotopes) represent sodium chloride salt clusters (Hao et al., 2001). The peaks at 270.825 represent $(\mathrm{NaCl})_{4} \mathrm{Cl}^{-}$. Peaks at 574.758 amu probably represent another salt cluster, $(\mathrm{NaCl})_{9} \mathrm{Cl}^{-}$, with a water molecule, like salt clusters with organic solvent molecules (Zhou \& Hamburger, 1996). The peaks at 632.7 amu have one more $\mathrm{NaCl}$ (i.e. $58 \mathrm{amu}$ ) than the peaks at 574.758 amu.

2-rhamnose-ribitol, as proposed in Fig. 3(a). The two glucose residues were labelled 1 and 2 for clarity.

To determine the linkages between the residues of 6X1 PS, we examined the periodate-treated $6 \mathrm{X} 1$ repeating units with MS-MS as we have done for 6C PS (Park et al., 2007b). This showed that ribitol and glucose 1 are cleaved by periodate, while glucose 2 and rhamnose are not. The masses of daughter ions showed that the phosphodiester bond is made to the second position of glucose 1 and that all other glycosidic bonds are the same as in 6B PS (data not shown). The MS-MS studies supported the proposed structure shown in Fig. 3(a).

During the alkali hydrolysis experiments for MS, we observed that the 6X1 PS was very resistant to alkali hydrolysis. To measure resistance to hydrolysis, we examined the ability of $6 \mathrm{~A}, 6 \mathrm{~B}, 6 \mathrm{C}$ and $6 \mathrm{X} 1 \mathrm{PSs}$ to inhibit binding of Hyp6BM8 (for 6B and 6X1 PSs) or Hyp6AG1 (for 6A and 6C PSs) to target PS after alkali hydrolysis for various time periods. $6 \mathrm{~A}$ and $6 \mathrm{C}$ PSs completely lost their ability to inhibit after only $1 \mathrm{~h}$ of hydrolysis. However, 6B PS lost $90 \%$ of its inhibitory ability in $8 \mathrm{~h}$ and more than $100 \mathrm{~h}$ of hydrolysis was needed for 6X1 PS to lose $90 \%$ of its inhibitory ability (Fig. 4). Thus, 6X1 PS is much more resistant than $6 \mathrm{~A}$ and $6 \mathrm{C}$ PS to alkali hydrolysis, and may be more resistant than $6 \mathrm{~B}$ PS.

\section{X1 is serologically similar to, but distinct from, 6B}

When the serological properties of TIGR6X1 were examined by the quellung reaction using polyclonal rabbit antisera, it was found to react with factor serum $6 \mathrm{a}$ and was typed as $6 \mathrm{~B}$. When we examined TIGR6X1 PS for binding various $\mathrm{mAbs}$ to $6 \mathrm{~A}$ and $6 \mathrm{~B}$ PS using an inhibition ELISA, we found it to be reactive with many mAbs to $6 \mathrm{~B}$ PS. For instance, TIGR6X1 inhibited Hyp6BM8 binding to 6B PS. These observations clearly demonstrated that $6 \mathrm{X} 1 \mathrm{PS}$ is serologically very close to 6B PS. However, we also found a $\mathrm{mAb}$ specific to 6B PS (Hyp6BM7) but not reactive with 6X1 PS (Fig. 5). Thus, 6X1 PS is serologically distinct from 6B PS.

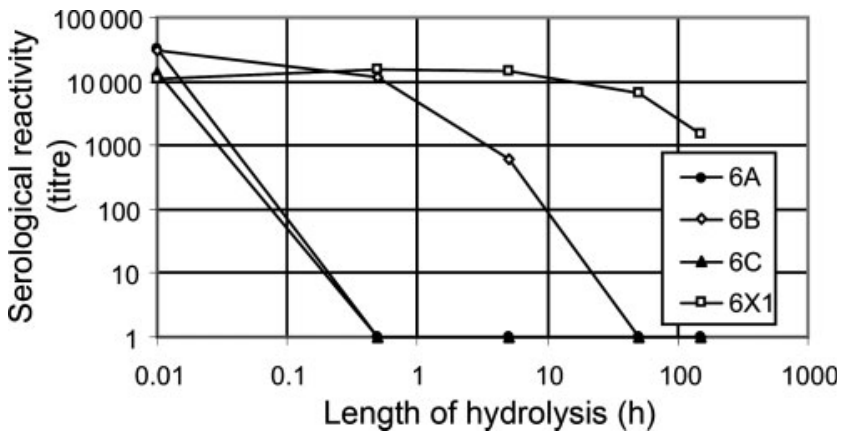

Fig. 4. Ability of various capsular PSs $\left(2 \mathrm{mg} \mathrm{m}^{-1}\right)$ to inhibit binding of $\mathrm{mAb}$ to ELISA plates ( $y$ axis) after the PSs were hydrolysed for various time periods ( $x$ axis); 'titre' indicates the dilution of a sample necessary to inhibit binding by $50 \%$. For $6 \mathrm{~A}$ and $6 \mathrm{C}$ PSs, ELISA plates were coated with $6 \mathrm{~A}$ PS and $\mathrm{mAb}$ Hyp6AG1 was used. For $6 B$ and 6 X1 PSs, ELISA plates were coated with $6 \mathrm{~B}$ PS and mAb Hyp6BM8 was used. 

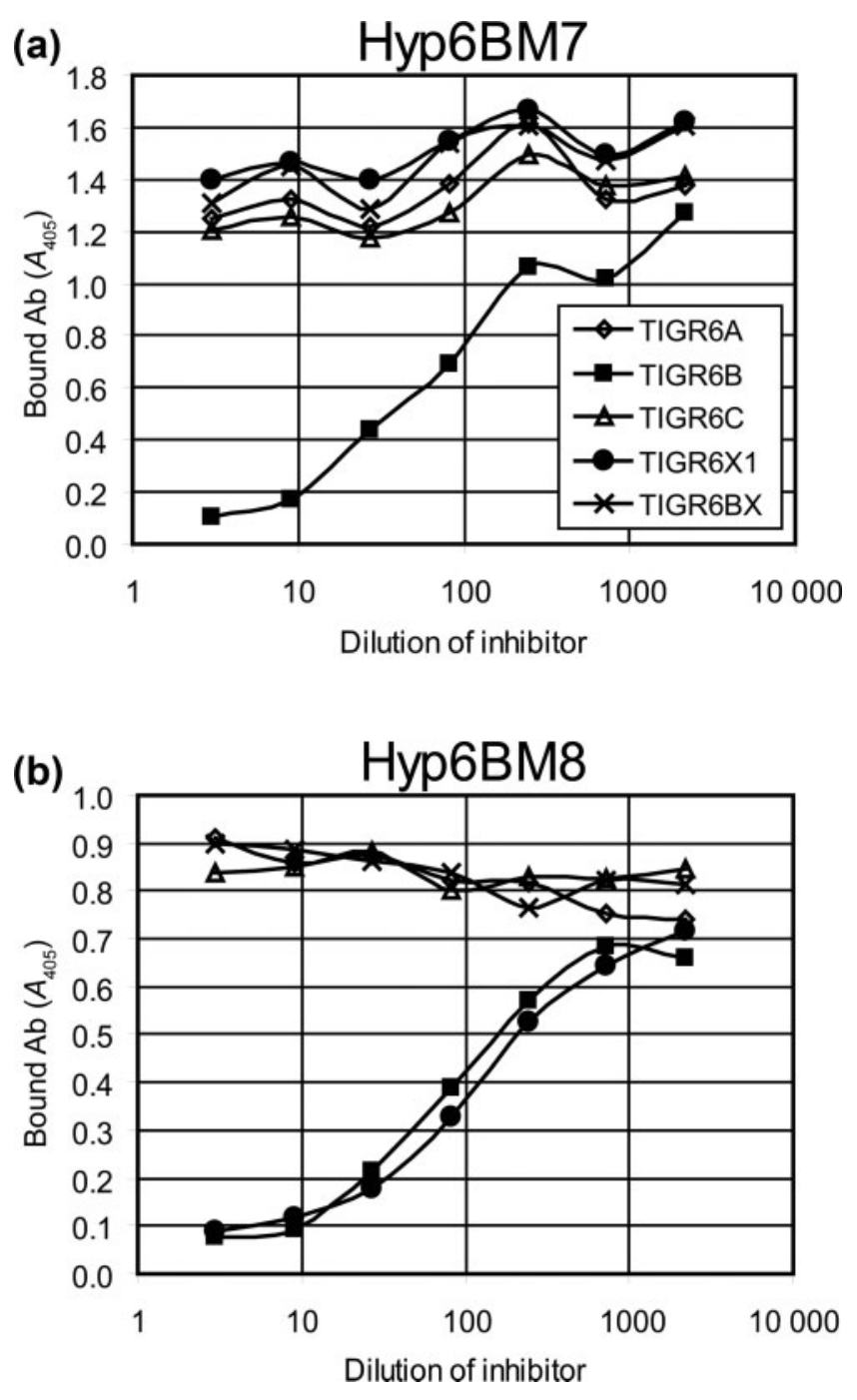

Fig. 5. Ability of lysates of various pneumococcal strains to inhibit the binding of mAbs Hyp6BM7 (a) and Hyp6BM8 (b) to 6B PScoated ELISA plates. The amount of $m A b$ bound ( $y$ axis) is shown in the presence of varying amounts of inhibitors ( $x$ axis). Lysates of pneumococcal strains were used as inhibitors. The pneumococcal strains are TIGR6A $(\diamond), \operatorname{TIGR6B}(\mathbf{\square}), \operatorname{TIGR6C}(\triangle), \operatorname{TIGR6X1}(\bullet)$ and TIGR6BX $(\times)$. TIGR6BX does not produce capsular PS.

\section{Pneumococcal isolates expressing serotype $6 \times 1$ PS were not found in nature}

The serological studies described above showed that if pneumococcal isolates expressing 6X1 PS are present in nature, they would have been typed as serotype 6B. To look for the presence of serotype 6X1 isolates in nature, we examined 264 pneumococcal isolates that had been previously typed as serotype $6 \mathrm{~B}$ using an inhibition ELISA capable of distinguishing between the $6 \mathrm{~B}$ and $6 \mathrm{X} 1$ serotypes (Fig. 5). These 6B isolates came from six continents [North America (109), South America (94), Europe (24), Asia (17), Africa (12) and Australia (8)], and were isolated from patients with bacteraemia (38), meningitis (19), pneumonia (40) and otitis media (17), as well as from healthy carriers (44) (106 isolates were from patients for whom the diagnosis is unknown). None of the $2646 \mathrm{~B}$ strains exhibited the antibody-binding profile of $6 \mathrm{X} 1$. Thus, the prevalence of serotype $6 \mathrm{X} 1$, if it exists, is much less than that of $6 \mathrm{~B}$.

\section{DISCUSSION}

The actual synthesis of the capsular PS requires cooperation among many different gene products. For instance, a new repeating unit made by a new glycosyltransferase must be compatible with the existing flippase as well as the polymerase before it can be expressed as a new capsule. Thus, to show that serotype $6 \mathrm{X} 1$ is possible, we produced a 'TIGR6X1' strain by inserting $w c i N_{6 C}$ into a $6 \mathrm{~B}$ capsule gene locus, and demonstrated that the new strain produces a capsular PS with the predicted structure, displays serological similarity to $6 \mathrm{~B}$, and can grow as well as other members of serogroup 6 in various growth conditions. Thus, serotype 6X1 could exist in nature.

Just as $6 \mathrm{C}$ was previously typed as ' $6 \mathrm{~A}$ ' by the classical typing method (Lin et al., 2006; Park et al., 2007b), the quellung reaction method typed the new $6 \mathrm{X} 1$ strain as serotype $6 \mathrm{~B}$. Thus, to identify natural isolates expressing serotype $6 \mathrm{X} 1$, we re-examined the isolates that had been classically defined as $6 \mathrm{~B}$ using mAbs. Despite our testing more than 250 such isolates, we did not find 6X1 isolates in nature. Additionally, wci $N_{6 C}$ was not detected among the $\mathrm{CDC}$ isolates that were serotyped as ' $6 \mathrm{~B}$ ' with the classical typing method (Dr B. Beall, CDC, personal communication). Thus, pneumococcal isolates expressing serotype 6X1 may not exist in nature. If serotype 6X1 exists in nature, its prevalence is extremely low.

Despite the fact that it is not detected in nature, the $6 \mathrm{X} 1$ serotype could emerge in nature by one of two mechanisms. One mechanism involves a mutation of the wciP gene of $6 \mathrm{C}$, since the only difference between $6 \mathrm{~A}$ and $6 \mathrm{~B}$ serotypes appears to be one nucleotide in the wciP gene. The mutation rate for pneumococci is $\sim 1 \times 10^{-8}$ (del Campo et al., 2005; Gould et al., 2007; Morosini et al., 2003) and a chronic obstructive pulmonary disease (COPD) patient with stable pneumonia may have $2.6 \times 10^{8}$ c.f.u. of pneumococci per millilitre of sputum (Sethi et al., 2007). Thus, the correct mutation should arise in almost all cases of 6C pneumonia and often in other 6C infections with lower bacterial load. The alternative mechanism involves the lateral gene transfer of wciN from a $6 \mathrm{C}$ strain into a $6 \mathrm{~B}$ strain as we have done here. This situation could actually occur in nature, since carriage of multiple pneumococcal serotypes can be relatively high among children (Gratten et al., 1986; Hill et al., 2008) and serotypes $6 \mathrm{~B}$ and $6 \mathrm{C}$ are fairly common in some parts of the world (e.g. Brazil) (Lin et al., 2006; Park et al., 2007a). Furthermore, homologous recombination would occur easily, since the $17 \mathrm{~kb}$ capsule gene loci of $6 \mathrm{~B}$ and $6 \mathrm{C}$ are 
almost identical except for the wciN gene. These considerations strongly suggest that the circumstances for creating serotype $6 \mathrm{X} 1$ exist in nature.

Given that the circumstances for creating $6 \mathrm{X} 1$ do exist in nature, it is interesting to consider reasons for its absence. It is possible that $6 \mathrm{C}$ could have appeared so recently that there may not have been enough time for 6X1 to appear. Alternatively, there could be natural immune barriers against $6 \mathrm{X} 1$, but we found that pre-immune human sera do not kill or opsonize TIGR6X1 (data not shown). The most likely explanation is that there has not been enough biological pressure to select $6 \mathrm{X} 1$ over $6 \mathrm{~A}, 6 \mathrm{~B}$ or $6 \mathrm{C}$. The need for selection pressure was recently demonstrated by an increase in the prevalence of serotypes $19 \mathrm{~A}$ and $6 \mathrm{C}$ following the use of the conjugate vaccine (Nahm et al., 2009; Park et al., 2008). In the absence of a survival advantage, the $6 \mathrm{X} 1$ serotype may have appeared in nature (as it should in almost every case of a 6C infection, as mentioned above) but has not been propagated as a consequence of competition with more abundant $6 \mathrm{~A}, 6 \mathrm{~B}$ or 6C serotypes. In an analogous manner, antibiotic-resistant strains survive and propagate when antibiotics are used clinically, and disappear when antibiotics are discontinued (Katsunuma et al., 2007).

6A PS was included in the original 14-valent PS vaccine, but it was replaced with 6B PS when the 23-valent vaccine became available in 1983, because 6B PS can elicit antibodies that cross-react with 6A PS and is much more resistant to hydrolytic breakdown than 6A PS (Zon et al., 1982). In this study, we also discovered that 6X1 PS is as chemically stable as 6B PS and much more resistant to hydrolysis than 6C PS. Since 6X1 PS would likely elicit antibodies cross-reactive with 6C PS, 6X1 may be more useful as a vaccine component than $6 \mathrm{C}$ PS.

As our knowledge of pneumococcal capsule genetics, biochemistry and serology advances, we may wish to produce pneumococcal strains that express artificially created capsular PS for various reasons. We propose that these artificially created strains should be named in a systematic manner to avoid their names being confused with those of natural strains. We chose to use ' $\mathrm{X}$ ' to denote such experimental strains. We chose ' $6 \mathrm{X1}$ ' as the name for our strain because this experimental strain belongs to serogroup 6 and this is the first experimental strain within serogroup 6 . This systematic approach should be applicable to any new strains created experimentally.

It is important to understand the evolution of the capsule gene locus, which encodes the most important virulence factor of pneumococci. Even when only two serotypes were known, the evolution of serogroup 6 was extensively studied (Mavroidi et al., 2004; Robinson et al., 2002). Serogroup 6 has become more interesting following the discovery of serotype 6C. Now, the serogroup would be even more interesting for evolution studies with a potential serotype, serotype $6 \mathrm{X} 1$.

\section{ACKNOWLEDGEMENTS}

The work was funded by a grant AI-031473 from the NIH and P.E. B. is supported by a training grant (T32 GM008111) from the NIH. UAB has applied for a patent covering the creation of $6 \mathrm{X} 1$. We thank Dr B. Beall and Mr R. Gertz at the CDC for assistance with the quellung reaction tests. We also thank Rob Cartee for performing GLC-MS and Marion Kirk for performing MS-MS analysis.

\section{REFERENCES}

Aanensen, D. M., Mavroidi, A., Bentley, S. D., Reeves, P. R. \& Spratt, B. G. (2007). Predicted functions and linkage specificities of the products of the Streptococcus pneumoniae capsular biosynthetic loci. J Bacteriol 189, 7856-7876.

Avery, O. T. \& Dubos, R. (1931). The protective action of a specific enzyme against type III pneumococcus infection in mice. J Exp Med 54, 73-89.

Cole, R. (1913). Treatment of pneumonia by means of specific serums. JAMA 61, 663-666.

del Campo, R., Morosini, M. I., de la Pedrosa, E. G., Fenoll, A., Munoz-Almagro, C., Maiz, L., Baquero, F. \& Canton, R. (2005). Population structure, antimicrobial resistance, and mutation frequencies of Streptococcus pneumoniae isolates from cystic fibrosis patients. J Clin Microbiol 43, 2207-2214.

Fedson, D. S. (1988). Pneumococcal vaccine. In Vaccines, pp. 271299. Edited by S. A. Plotkin \& E. A. Mortimer, Jr. Philadelphia, PA: W. B. Saunders.

Gould, C. V., Sniegowski, P. D., Shchepetov, M., Metlay, J. P. \& Weiser, J. N. (2007). Identifying mutator phenotypes among fluoroquinolone-resistant strains of Streptococcus pneumoniae using fluctuation analysis. Antimicrob Agents Chemother 51, 3225-3229.

Gratten, M., Gratten, H., Poli, A., Carrad, E., Raymer, M. \& Koki, G. (1986). Colonisation of Haemophilus influenzae and Streptococcus pneumoniae in the upper respiratory tract of neonates in Papua New Guinea: primary acquisition, duration of carriage, and relationship to carriage in mothers. Biol Neonate 50, 114-120.

Hao, C., March, R. E., Croley, T. R., Smith, J. C. \& Rafferty, S. P. (2001). Electrospray ionization tandem mass spectrometric study of salt cluster ions. Part 1 - investigations of alkali metal chloride and sodium salt cluster ions. J Mass Spectrom 36, 79-96.

Hausdorff, W. P., Bryant, J., Paradiso, P. R. \& Siber, G. R. (2000), Which pneumococcal serogroups cause the most invasive disease: implications for conjugate vaccine formulation and use. Part I. Clin Infect Dis 30, 100-121.

Henrichsen, J. (1995). Six newly recognized types of Streptococcus pneumoniae. J Clin Microbiol 33, 2759-2762.

Hill, P. C., Cheung, Y. B., Akisanya, A., Sankareh, K., Lahai, G., Greenwood, B. M. \& Adegbola, R. A. (2008). Nasopharyngeal carriage of Streptococcus pneumoniae in Gambian infants: a longitudinal study. Clin Infect Dis 46, 807-814.

Jiang, S. M., Wang, L. \& Reeves, P. R. (2001). Molecular characterization of Streptococcus pneumoniae type 4, 6B, 8, and 18C capsular polysaccharide gene clusters. Infect Immun 69, 1244-1255.

Kamerling, J. P. (2000). Pneumococcal polysaccharides: a chemical view. In Streptococcus Pneumoniae Molecular Biology and Mechanisms of Disease, pp. 81-114. Edited by A. Tomasz. Larchmont, NY: Mary Ann Liebert.

Katsunuma, Y., Hanazumi, M., Fujisaki, H., Minato, H., Hashimoto, Y. \& Yonemochi, C. (2007). Influence of avilamycin administration and its subsequent withdrawal on emergence and disappearance of 
antimicrobial resistance in enterococci in the intestine of broiler chickens. J Appl Microbiol 102, 1159-1166.

Lin, J., Kaltoft, M. S., Brandao, A. P., Echaniz-Aviles, G., Brandileone, M. C., Hollingshead, S. K., Benjamin, W. H. \& Nahm, M. H. (2006), Validation of a multiplex pneumococcal serotyping assay with clinical samples. J Clin Microbiol 44, 383-388.

Mavroidi, A., Godoy, D., Aanensen, D. M., Robinson, D. A., Hollingshead, S. K. \& Spratt, B. G. (2004). Evolutionary genetics of the capsular locus of serogroup 6 pneumococci. J Bacteriol 186, 81818192.

Morosini, M. I., Baquero, M. R., Sanchez-Romero, J. M., Negri, M. C., Galan, J. C., del Campo, R., Perez-Diaz, J. C. \& Baquero, F. (2003), Frequency of mutation to rifampin resistance in Streptococcus pneumoniae clinical strains: hexA and hexB polymorphisms do not account for hypermutation. Antimicrob Agents Chemother 47, 1464 1467.

Nahm, M. H., Lin, J., Finkelstein, J. A. \& Pelton, S. I. (2009). Expansion of the newly discovered pneumococcal serotype $6 \mathrm{C}$ in the nasopharynx following introduction of pneumococcal conjugate vaccine. J Infect Dis (in press).

Park, I. H., Park, S., Hollingshead, S. K. \& Nahm, M. H. (2007a). Genetic basis for the new pneumococcal serotype, 6C. Infect Immun 75, 4482-4489.

Park, I. H., Pritchard, D. G., Cartee, R., Brandao, A., Brandileone, M. C. \& Nahm, M. H. (2007b). Discovery of a new capsular serotype (6C) within serogroup 6 of Streptococcus pneumoniae. J Clin Microbiol 45, 1225-1233.
Park, S. Y., Moore, M. R., Bruden, D. L., Hyde, T. B., Reasonover, A. L., Harker-Jones, M., Rudolph, K. M., Hurlburt, D. A., Parks, D. J. \& other authors (2008). Impact of conjugate vaccine on transmission of antimicrobial-resistant Streptococcus pneumoniae among Alaskan children. Pediatr Infect Dis J 27, 335-340.

Robinson, D. A., Briles, D. E., Crain, M. J. \& Hollingshead, S. K. (2002). Evolution and virulence of serogroup 6 pneumococci on a global scale. J Bacteriol 184, 6367-6375.

Sethi, S., Sethi, R., Eschberger, K., Lobbins, P., Cai, X., Grant, B. J. \& Murphy, T. F. (2007). Airway bacterial concentrations and exacerbations of chronic obstructive pulmonary disease. Am J Respir Crit Care Med 176, 356-361.

Sun, Y., Hwang, Y. \& Nahm, M. H. (2001). Avidity, potency, and crossreactivity of monoclonal antibodies to pneumococcal capsular polysaccharide serotype 6B. Infect Immun 69, 336-344.

Tettelin, H., Nelson, K. E., Paulsen, I. T., Eisen, J. A., Read, T. D., Peterson, S., Heidelberg, J., DeBoy, R. T., Haft, D. H. \& other authors (2001). Complete genome sequence of a virulent isolate of Streptococcus pneumoniae. Science 293, 498-506.

Zhou, S. \& Hamburger, M. (1996). Formation of sodium cluster ions in electrospray mass spectrometry. Rapid Commun Mass Spectrom 10, 797-800.

Zon, G., Szu, S. C., Egan, W., Robbins, J. D. \& Robbins, J. B. (1982). Hydrolytic stability of pneumococcal group 6 (type 6A and 6B) capsular polysaccharides. Infect Immun 37, 89-103.

Edited by: M. Kilian 